\title{
Case Report: Those pesky dots. What are they?
}

\author{
Paul T. Rose, MD, JD, FISHRS I Miami, Florida, USA I paultrose@yahoo.com; Michael Morgan, MD I \\ Tampa, Florida, USA
}

\section{INTRODUCTION}

The follicular unit excision (FUE) harvesting process has continued to gain in popularity. The primary advertised value of this technique is that the surgery does not result in a linear scar.

As the harvesting technique involves punches of one type or another, a round hole is the result of the tissue removed. Hole sizes can vary from approximately $0.75 \mathrm{~mm}$ to $1.2 \mathrm{~mm}$ at the time of surgery. These holes are allowed to heal by second intention. The resulting defect is a disciform, often hypopigmented area of scar tissue. The defect is at times larger than the original punch diameter. Some advocates of the FUE technique have suggested in various forms of media and presentations that the technique leaves no visible scars and that the hypopigmented dots are not actually scars.

Here, we detail our observations of the histological appearance of removed tissue that included two of the "dots" in a patient who had undergone a prior FUE procedure.

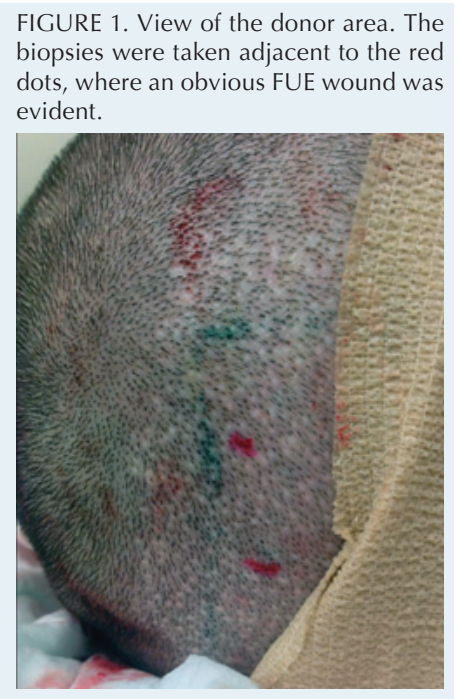

\section{CASE REPORT}

The patient was a 27-year-

old Hispanic male who previously had FUT (strip) in 2005 and then underwent an FUE procedure of 1,000 grafts in 2016 with roboticassisted surgery using an $18 \mathrm{~g}$ punch (Figure 1). One year after robotic-assisted FUE, the patient consented to the removal of two of the hypopigmented dots that resulted from his surgery.

Two hypopigmented circular areas consistent with the FUE harvesting sites were marked and then cleansed with betadine and alcohol. The areas were anesthetized with $1 \%$ xylocaine with epinephrine 1:100,000.

Using a $2 \mathrm{~mm}$ punch, the specimens were excised and placed in formalin. The tissue was then sent to a dermatopathologist. The tissue was stained with H\&E (hematoxylin and eosin), Trichrome, Reticulin, and Elastin stains. The results indicated that the tissue obtained was consistent with scar tissue (Figure 2).

\section{DISCUSSION}

This case report should serve to demonstrate that the wounds created by the FUE process result in scars. These scars are particularly evident when the entire follicular unit is removed, as they are void of hair. They are the "pesky" dots we observe after FUE healing takes place.
FIGURE 2. Histology: Note the presence of dense pink (organizing) collagen juxtaposed to and above the follicle and punctuated by an arcuate array of capillaries. The latter features in particular, the location and vessel density should alert the microscopist to the possibility of a scar associated with a previous procedure (transplant) in lieu of the typical intrafollicular stelae that accompanies catagen/telogen follicles or broad interfollicular scarring seen in conjunction with scarring alopecia.
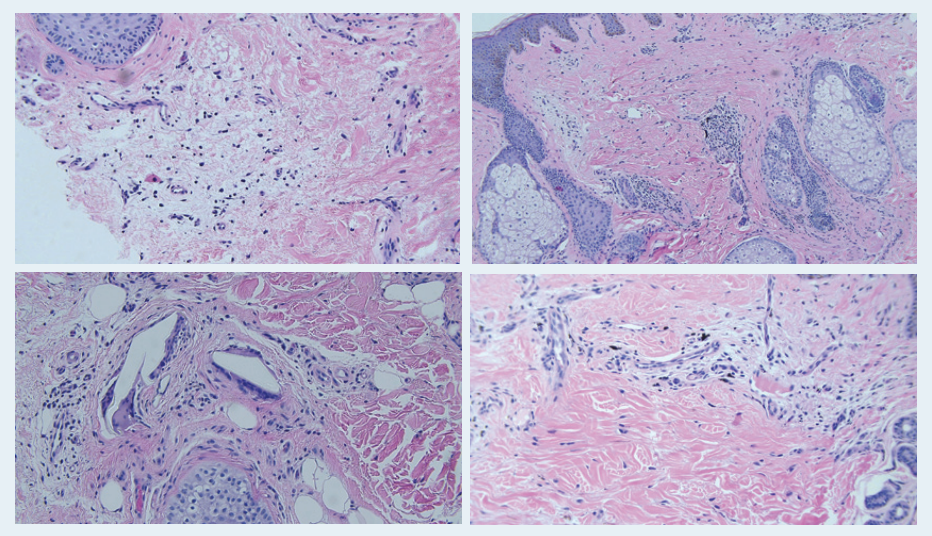

As the surgeon harvests subsequent grafts and some grafts are obtained adjacent to one another, there is the creation of prominent visible scarring due to the increased area of bald skin. As more grafts are removed, there may be thinning of the donor area. As thousands of FUE grafts are harvested, one can reason and calculate that the area of scarring from FUE could easily exceed that of a linear scar.

Some critics of the pathology report may point to the use of the robot that, at that time, was using an $18 \mathrm{~g}$ needle, and argue that the robotic-assisted surgery made bigger defects than a surgeon would using a $0.9 \mathrm{~mm}$ or smaller punch. However, we would point out that we have observed the same scar defects in patients who have undergone FUE with smaller punches. It should be emphasized that it is not unusual for the diameter of FUE scars to be larger than the original punch diameter. We attribute this to a lack of contractile forces on the tissue when a great many punches are made in the donor area. 